\title{
A Survey on Cloud Computing for Information Storing
}

Sathiyasheelan Ravichandran

Faculty of Business Informatics, Riga Technical University, Riga, Latvia.

**Corresponding Author: rsathiya2196@gmail.com

Received: 17.06.2020, Revised: 10.07 .2020 , Accepted: 10.08.2020, Published: 31.09 .2020

DOI:

10.53409/mnaa.jcsit20201202

Abstract: Cloud computing is a technique for storing the information virtually. It may comprise of database, storage, tools, servers, networking and software services. It deals with virtual storing and retrieving of data from anywhere by the help of internet. This paper uses cloud computing technique in education for uploading study materials, videos, sharing information to the students and for conducting tests. The symmetric key encryption technique is used in this concept, where one key is utilized for both decryption and encryption. The advanced encryption standard algorithm (AES) was used for securing the data in the cloud, where it gives high security and faster execution time. This technique is mainly based on improving the concept of virtual classroom by using cloud computing.

Keywords: AES algorithm, cloud computing, E-learning, storage, virtual classroom

\section{INTRODUCTION}

r loud computing is a biggest platform for all services providers over the internet. The main resources of the cloud provide storage, server, database, networking and software for all the tools and applications. Cloud is a major platform for the educational field for online learning, smart technology and networking, for each process of services and local storage devices, hard drive it will report the database for the verification. By using cloud services for a college management, business process, marketing fields. The reason for cloud computing option for people and business including cost saving, increased the product productivity, speed and efficiency automatically increases, performance of the services in all platform, security is highly effective by using different algorithm based on the applications. In cloud computing the information is accessed virtual space with the help of remote. Business aspects of all companies provide cloud service user can enable to save applications and files on remote servers and then access every data through internet.

In recent years, cloud computing has demonstrated a variety of points of concern over traditional figuring models to be mainstream. Average desirable conditions with mobility, the convergence of adaptability, energy conservation, and cost savings. Cloud computing has been introduced as a general term for describing a set of advanced on-demand services for computing offered by commercial providers like Google, Amazon, and Microsoft. It means a computing infrastructure model is considered as a "cloud," from which organizations, industries, and people accesses applications on demand across the world. The fundamental concept beneath this technology was providing storage, computing, and software "as a service". National Institute of Standards and Technology (NIST) describes Cloud computing as a "model for enabling universal, convenient, and on demand access of network to configurable computing resources in a shared pool which could be quickly provided and delivered with minimum managerial efforts or service provider interactions".

Cloud is an on-demand advance model for the IT world, constantly taking into account simulation and cloud computing developments. Cloud computing involves both the applications represented as services through Internet and hardware and software of the device used in the data center to offer the service. The systems 
themselves were known as IaaS (Infrastructure as a Service), Software as a Service (SaaS), and PaaS (Platform as a Service) for describing their service.

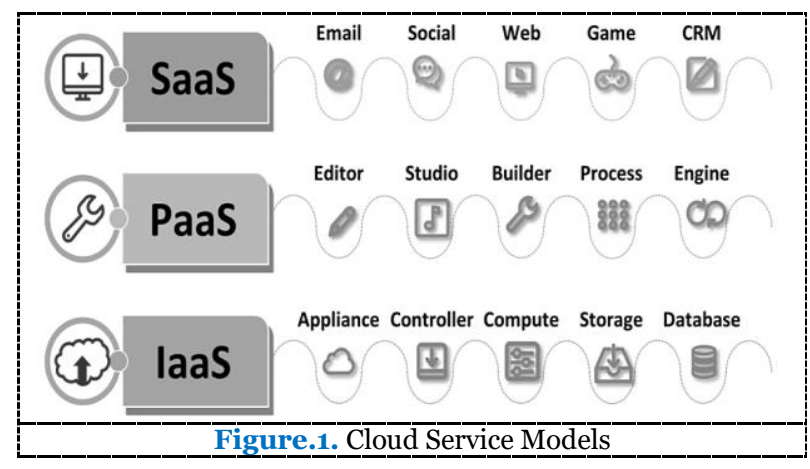

Cloud computing process data from the devices from around the all storage of data based on the virtual platform services providers. The internet cloud services data and application and work are accessible from any devices with could link to the internet. Cloud computing have different types of user can approaches the cloud services like public cloud, private cloud and hybrid cloud. Public cloud service provides their service to the user through the internet for free. Private cloud service provides their service to the business aspects of companies for certain number of people. The combination of public cloud and private cloud of both elements are known as hybrid cloud, these services provide system of network that supply hosted services.

Although there are no official recommendations for the use of cloud-based systems in the education field, an inquiry to define and establish a reliable platform as a structured method in higher education is desirable as well as an area of interest and study. There is no question that teaching and learning strategies focused on personal computer equipment may pose a serious risk of exacerbating the divide among students who have access to different resources and technologies and students who cannot endure the same.

\section{RELATED WORK}

\subsection{Cloud Computing - An Advanced E- Learning Platform of School Education}

Cloud computing trends are use to develop the e-learning services. A platform of e-learning is fully concentrated on student education system and their knowledge of the upcoming technologies. The e-learning system estimate the need of new platform that are depend upon the techniques like mobility, flexibility, individualization availability, and openness of education. Cloud technology are used in facilities and computer resources are available on the web service user like platform as a services, software as a services, hardware as a services, infrastructure as a services, communication as a services. The user accesses the online cloud computing by using certain equipments like laptop, net book, and smart phone. The advantages of these techniques reduce the hardware requirements and cost. Implementation of cloud computing technology is developing the modern education system it can use the main concepts of e-learning techniques to learn the current technology for improving the education resources.

\subsection{E-Learning in a Cloud Computing Environment}

Cloud is a growing technology in every sector the storage services is include based on the different types of services and the main goal of the cloud to develop the many education institutions with high ability for infrastructures and resources to execute the e-learning system. The concept of the paper explains the advantages and limitation of the cloud e-learning. Cloud computing is the growth future e-learning across institutions of worldwide services. Educational institution is focusing on offering students, faculties and administrations with capability to improve the modern mobile world.

\subsection{The Application of Cloud Computing In Education Informatization}

The application research of cloud computing in educational information, the tradition of technologies based on computers include the network storage, virtualization, distributed computing play a major role to share the current status of the cloud service, parallel commuting techniques is used to delivered the information on the same time to the users, network technology is a main process for sharing a data with fast and secure manner and automation techniques is initially the process is start until the last the same process. The proposed method of cloud computing is used by IBM and GOOGLE platform. The cloud computing was significance for developing information technology in field of education. The cloud is a convenient and cheap for more data processing 
of modern education system. The level of education technology will be enhanced the information sources in future.

\subsection{A Proposed Model for Education System using Cloud Computing}

Education and cloud computing combine to give a quality type of education under the information technology develop the economic growth. The faculties are ready to develop an informatics future student under the backbone of cloud services providers like sales force. The information sharing techniques make a student's more knowledgeable atmosphere. Many services providers offer various cloud based application for user who can work simply for academia of cloud computing. The e-learning system facing teaching challenges of cloud computing. The propose model of cloud computing the teachers and students could share the course programs through the cloud and students can update their examination, materials and assignments. The attendance records are also stored in cloud services. Cloud computing education was to minimize the difficulties in teaching infrastructure.

\subsection{E- Learning Based on Cloud Computing Technology}

Cloud computing is a growing rapidly, with application including the area based on education. The telecommunication devices like desktop, laptops, tablets, mobile, music players it make a product to distributed compare to any centralized entity. E-learning system is usually require many hardware and software resources offer the cloud computing facilities to achieve flexibility, efficiency for good process e- learning services. Cloud e-learning is an approach for selecting the cloud as it promise very clear advantages. Cloud support digital services for storage data to distributed the access centralized system uses of application and resources for several cloud applications. By using the cloud platform is less amount of cost and the data sharing under safe and secure process of cloud services providers increase the learning facilities.

\subsection{AES Algorithm Based Approaches}

This segment highlights the significance of advanced encryption standard (AES) technology, as it is the key algorithm method used in the conducted study of both the encryption process and the decryption process. A variety of papers was evaluated on the basis of a testing methodology in which the AES algorithm is used in a number of cloud services related applications in which other algorithms are effectively compared.

Abha Sachdev and Mohit Bhansali (2013), with the monumental growth of sensitive cloud data, cloud protection has become more critical than any time in the modern past. Cloud information and software live in highly adaptable storage systems and can be accessed all over the world. Unfortunately, the advancement of cloud clients was combined with the emergence of malicious behavior in the cloud. An ever-increasing number of bugs have been discovered and new security alerts have been regularly issued. A vast number of clients access the Cloud for diverse reasons and thus need incredibly safe and resourceful services. The development of the cloud, especially in the increasing scope of apps, includes far more protection and validation.

Roshani Raghatate et al., (2014), Adding AES to information security provide benefits of less memory usage and less time to measure as compared to alternative algorithms. About the fact that cloud infrastructure has its own security features, the client should select architecture depending on its security. Each cloud service has its own set of rules, pricing, capability, support as well as other essential features. The primary consideration dealt with in this proposal was the encryption concept for protecting data by making it inaccessible to everyone.

A.M Abdullah, (2017), AES algorithm was one of the familiar and commonly utilized symmetric block cipher algorithms used worldwide. This algorithm got the individual basic framework for encrypting and decrypting confidential data and was utilized in hardware and software worldwide. It was very challenge for hackers to acquire the original data when encrypted with an AES algorithm. No proof was there to date that this process of algorithm could be solved. AES could accommodate three different sizes of keys, such as AES 128, 192, and 256 bits, and both of these 128-bit block sizes.

\section{CLOUD SERVICE MODELS}

Cloud services are provided as IaaS, PaaS, and SaaS. It is a combination of many techniques, comprising distributed and grid computing, and 
service delivery network by using Internet. The public cloud platform is very complicated when contrasted with a conventional data center environment. Based on the model of Cloud computing, an institution or organization gives up direct access to significant features of security, provides a higher level of trust over the Cloud provider. The three different types of service models in cloud computing are:

\subsection{IAAS}

Infrastructure as a service (IAAS) is a virtual resources that includes virtual servers, networks, processing power, hardware and storage. These services can be accessed by cloud computing platform based on pay as per usage of services. This service reduces the cost and complexity of buying the software and managing the servers rather than using as we need. The services are highly scalable and flexible and also maintain the system backup and developing measures.

\subsection{PAAS}

Platform as a service (PAAS) was a cloud computing service model which provides storage, database management system, hardware, networking, servers, middleware, software, operating system, application framework, programming languages and other development tools to users for creating applications. These services can be easily accessible by cloud platform services based on pay for usage. This is highly scalable and it makes easy for the customers to create, run, manage, test and deploying the web applications.

\subsection{SAAS}

Software as a service (SAAS) was an on-demand software that provides software to the users by accessing through internet. This service is cost effective and the users no need to pay money for purchasing and installing the software because it works on monthly subscription of software. The software is provided by third party vendors as a host application to the customers based subscription of products. This model can be used in business applications, customer relationship management, document management, social networking and mail services.

\subsection{Selection of cloud deployment model}

A cloud computing based solutions can be used in following ways,
Community Cloud: This uses a framework with network for its assurance just like a grid.

Public cloud: This kind of solution is given by the Google, amazon where the data must be ensured in a private framework with an assurance that it is ensured there also it relies upon pay start and demands more prominent security from a broad amounts of vindictive groups.

Private Cloud: This kind of feature is required in private affiliations and government firms, and besides where the data stored needs more care and should be managed carefully. These can develop their own particular plan of standards in a cloud where simply the administrators of that affiliation can get to.

Hybrid Cloud: It is mix of the two clouds described above i.e. Private cloud and Public cloud.

Cloud computing has been used in a variety of applications globally. The research work was in the domain of cloud computing, for which technology was necessary to be used for technological development in the education application. Several companies or organizations now have minimum one program or half of their computing technology over the cloud. Cloud environments are ripening and, in few cases, getting more nuanced. Although 43 percent use just hybrid cloud and 12 percent just use multi cloud, 30 percent use both.

\section{SYSTEM DESIGN}

The architecture diagram (see fig 1) explains the concept of cloud computing based virtual classroom technique. This system helps to improve the communication between the students and the staff by storing and retrieving all the information from the cloud. The cloud computing contains all the details of staffs, students, study materials, videos and institution related information. Here, the cloud is connected with staff and students, where staff is responsible for uploading study materials, videos, conducting tests, viewing student performance and sharing general information among the students. Students are responsible for taking tests from anywhere, viewing videos, materials and information, communicating with 
friends and can do the project by the use of cloud computing.

Cloud computing based virtual classroom technique makes the students to gain knowledge from anywhere and from any device. It helps the staff to manage more number of students in a single time and can view the student's performance from anywhere and from any device. This technique does not need any storage device for storing the details, where it has the capacity of storing the information virtually in cloud. This paper is based on symmetric key encryption, it contains single key for both encryption and decryption techniques. The algorithm used for this technique is advanced encryption standard algorithm (AES). AES is commonly typically used for cloud protection. AES was proposed in order to replace DES in functional implementations. AES was adopted by the NIST on 26 November 2001. AES was a symmetric-key algorithm that means that the single key was used for both data decoding and data encoding. AES was also called RIJNDAEL, named after the names of its inventors John Daemen and Vincent Rijmen. AES was unpredictable and depended on the duration of the key. AES used 10 series for 128-bit keys, 14 series for 256-bit keys and 12 series for 192-bit keys. Both of the series uses an alternative 128bit series key, which was predicted from the original AES key. AES algorithm provides high security, allows less space and quicker execution time than other algorithms. This algorithm encrypts and decrypts data using cryptographic bit keys. This guarantees high protection for data in the cloud world.

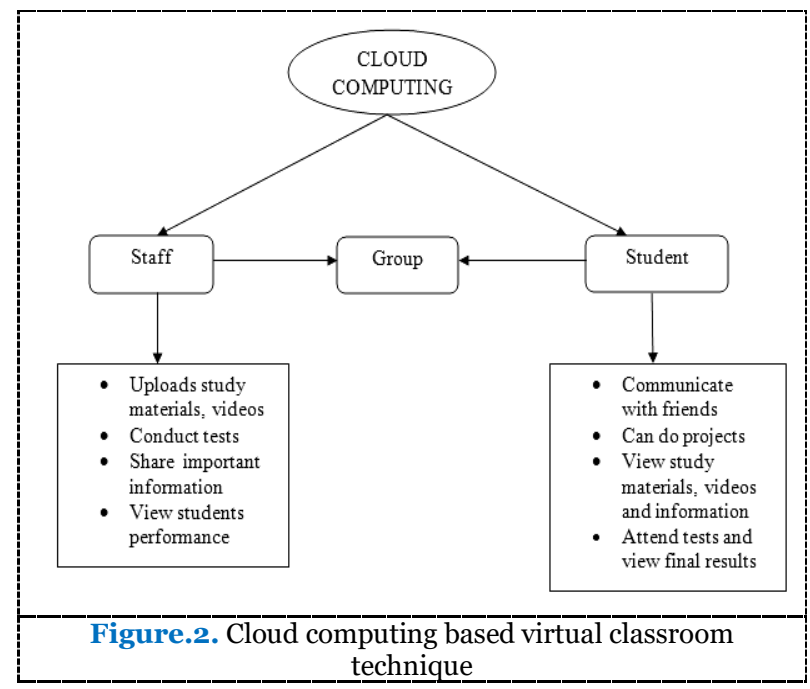

AES works well in both the software and hardware processes under a wide range of circumstances. This includes 8-bit and 64-bit systems and DSPs. Its underlying parallelism enables the optimal use of processor resources contributing to execution of large-scale programming. This algorithm got fast key configuration time and improved key operation. It needs less memory for use, making it suitable for small space scenarios. The structure had a tremendous ability to benefit from parallelism at the stage of teaching. There were no critical bad keys to AES. It supports both block size and key size which were 32 multiples (more notable than 128-bits). A numerical measurement of the ciphertexts was not possible despite a huge number of experimental had been carried out. No differential and linear cryptanalysis attacks on AES have yet been developed.

Proper convergence of face-to-face and interactive learning is needed to ensure the continuity of higher education through the use of digital skills. A proper balance of digital life and physical life will yield enormous benefits for the economy and society. Future learning education would focus more on artificial learning, virtual machines/online computers, or cloud-based information storage. Real-life and virtual learning will complement one another, and consumers would have remote access to apps independent of time and physical spaces.

Transition to virtual learning was an interim solution to the coronavirus pandemic. What is needed is rethinking, creativity, automation, and quality management, with an emphasis on learning digitalization. The design of the layout of the courses and the mode of delivery in higher education must be based on scenario planning. Instructional design is a common concept coined in virtual learning that ensures online learning is most successful. This examines the ability to apply modern technologies to the immersive and innovative implementation of e-classes. To sum up, successful digital learning involves imagination, ingenuity, and the design of innovative technologies to involve students before and during lectures on the digital platform. A constructive strategy is required to share the expertise and experience of workers with varied needs. 


\section{CONCLUSION}

Cloud computing based virtual classroom technique is mainly based on improving the communication between the staff and the student by storing and retrieving all the information's from cloud. Cloud computing helps the people to store large amount of data virtually and allows the people to retrieve the data from anywhere and by any device. Advanced encryption standard (AES) algorithm is used in this concept, where it provides high security, faster execution time, and high encryption capacity and requires low amount of memory usage. This system helps all the educational institution for storing, retrieving and viewing all the students and staffs performance virtually by using cloud computing technology.

\section{ETHICS APPROVAL AND CONSENT} TO PARTICIPATE

Not applicable.

\section{HUMAN AND ANIMAL RIGHTS}

No animals/humans were used for studies that are basis of this research.

\section{CONSENT FOR PUBLICATION}

Not applicable.

\section{AVAILABILITY OF DATA AND MATERIALS}

The authors confirm that the data supporting the findings of this research are available within the article.

\section{FUNDING}

None.

\section{CONFLICT OF INTEREST}

The authors declare no conflict of interest, financial or otherwise.

\section{ACKNOWLEDGEMENTS}

The author would like to thank Faculty of Business Informatics, Riga Technical University for providing support while carrying out this work.

\section{REFERENCES}

[1] Ishaq A. and Brohi M. N. Cloud Computing in Education Sector with Security and Privacy Issue: A Proposed Framework. International Journal of Advances in Engineering \& Technology vol. 8, no. 6, pp. 889-898.

[2] Engr. Ali Ahmed, Huma Ali Ahme. A Proposed Model for Education System Using Cloud Computing.

[3] Hongyu Pei Breivold and Ivica Crnkovic. (2014). Cloud Computing Education Strategies. 978-1-4799-4970-0.

[4] Majid Shirzad, Ali Hoseinpanah, Mehdi Ahmadipour, Hojat Rahimi. (2012). E-Learning Based on Cloud Computing, of20121ntemational of Cloud Computing, Technologies, Applications \& Management 978-1-46734416-6.

[5] Bo Wang, HongYu Xing. (2015). The Application of Cloud Computing in Education Informatization, 978-14244-9763-8.

[6] Shaoyong Chen, Min Lin, and Huanming Zhang. (2019). Research of mobile learning system based on cloud computing. International Conference on e-Education, Entertainment and e-Management, 978-1-4577-1382-8.

[7] Utpal Jyoti Bora, Majidul Ahmed. (2013). E learning using Cloud Computing International Journal of Science and Modern Engineering (IJISME) ISSN: 23196386, Volume-1, Issue-2.

[8] Shyshkina Mariya. (2016). Cloud computing - an advanced e-learning platform of school education, 14th International Conference on Interactive Collaborative Learning, 978 -1-4577-1747-5.

[9] Mohammed Ketel. (2014). E-Ieaming in a Cloud Computing Environment, 978-1-4799-6585-4.

[10] Mohssen M. Alabbadi. (2011). Cloud Computing for Education and Learning: Education and Learning as a Service (ELaaS), 14th International Conference on Interactive Collaborative Learning, 978 -1-4577-1745.

[11] Roshani Raghatate, Sneha Humne, and Roshna Wadhwe. (2014) A Survey on Secure Cloud Computing using AES Algorithm, IJCSMC, Vol.3, Issue.12, pg.295 $-301$.

[12] Abha Sachdev and Mohit Bhansali. (2013) Enhancing Cloud Computing Security using AES Algorithm, International Journal of Computer Applications (0975 - 8887) Volume 67- No.9.

[13] Ako Muhamad Abdullah. (2017). Advanced Encryption Standard (AES) Algorithm to Encrypt and Decrypt Data, Cryptography and Network Security. 